\section{The combined effect of simulated microgravity and radiation on chromosome aberrations in human peripheral blood lymphocytes}

\author{
Sakuya Yamanouchi ${ }^{1}$, Takuya Adachi ${ }^{1}$, Yukari \\ Yoshida ${ }^{1}$, Jordan Rhone ${ }^{2}$, Jian-Hua Mao ${ }^{3}$, Keigi \\ Fujiwara $^{4}$, Premkumar B. Saganti ${ }^{2}$, Akihisa \\ Takahashi $^{1, \star}$, Megumi Hada ${ }^{2, \star}$
}

${ }^{1}$ Gunma University Heavy Ion Medical Center, Maebashi, Gunma 371-8511, Japan

${ }^{2}$ Radiation Institute for Science \& Engineering, Prairie View A\&M University, Prairie View, TX 77446, USA

${ }^{3}$ Biological Systems \& Engineering Division, Lawrence Berkeley Laboratory, Berkeley, CA 94720, USA

${ }^{4}$ Department of Cardiology, University of Texas MD Anderson Cancer Center, Houston, TX 77030, USA

\section{Abstract}

To understand the combined effect of microgravity $(\mu G)$ and space radiation on cells is an important requirement for the anticipated space travel by humans. Cultured cells, such as human fibroblasts and lymphoblasts, were used in previous studies, and we observed changes in the expression of some cell-cycle-related genes and increased chromosome aberrations (CAs) in these cells under simulated $\mu G$. In this study, we investigated the combined effect of $\mu G$ and space radiation on human peripheral blood lymphocytes. Whole blood was irradiated with X-ray or carbon-ion (C-ion) beam while being exposed to simulated $\mu G$ using a threedimensional clinostat. The frequency of CA was assessed using the three-color fluorescence in situ hybridization on chromosome spreads of colcemid-induced prematurely condensed chromosomes in lymphocytes during the first cell division post irradiation. Compared with the cells irradiated at $1 G$, the frequency of $C A$ was increased in cells simultaneously exposed to simulated $\mu G$ and radiation even though cells were irradiated by the same doses $(0.5-1.0 \mathrm{~Gy})$. This result is similar to those of our previous studies in which we used human lymphoblast

Received: April 27, 2021; Accepted: July 8, 2021

*To whom Correspondence should be addressed:

Tel.: +81-27-220-7917; Fax: +81-27-220-7919;

E-mail: a-takahashi@gunma-u.ac.jp

Tel.: +1-936-261-3155; E-mail: mehada@pvamu.edu
TK6 cells and human fibroblast 1BR-hTERT cells. These ground-based experiments give insights into the biological effects of the space environment where radiation and $\mu G$ coexist. @2021 Jpn. Soc. Biol. Sci. Space; doi:10.2187/bss.35.15

\section{Introduction}

Space radiation is a major environmental factor whose biological effects must be neutralized, minimized, and/or reversed for the crew of all space missions. For this reason, space radiation biology has been an active area of research for many years. Space radiation can be classified into two categories: solar particle rays, which are suddenly generated by perpetually-occurring explosions on the solar surface, and high-energy galactic cosmic rays, which originate from supernova explosions and shower the entire universe. These rays range from low-linear energy transfer (LET) radiation, such as electrons and protons, to various types of heavy-ion beams (Furukawa et al., 2020; Takahashi et al., 2018). The latter types are high-LET radiation, generate more DNA double-strand breaks (DSBs) (Li, 2007), and are expected to cause more serious biological consequences. However, there are only a limited number of studies on cells exposed continuously to space radiation at low doses and low dose rates. To directly estimate biological effects of space radiation, chromosome damages in peripheral blood lymphocytes is the most widely used biomarker.

The fluorescence in situ hybridization (FISH) method is a standard technique for gene mapping (Lichter et al., 1990; Pinkel et al., 1986) in which two or three chromosomes are targeted for staining. Compared with a simple Giemsa staining (Edwards and Lloyd, 1991; Sabatier et al., 1987), this method is more effective in identifying complex exchanges because different chromosomes can be identified by color coding (Anderson et al., 2000; Durante et al., 1999; Griffin et al., 1995). The FISH method has a higher resolution for the locations and orders of genes (Ferguson-Smith, 2015) than classical methods for showing genetic linkage based on recombination data from pedigree analysis. The multicolor FISH method has been developed to stain all 23 pairs of human chromosomes (Loucas et al., 2001). Each chromosome is chromatically identified using a combination of five fluorescently-tagged probes, and chromosome aberration (CA) can be identified through just one hybridization (Schröck et al., 1996).

To assess the effects of space radiation on returning astronauts, CAs in their peripheral blood lymphocytes have been used as an indicator. In one study, peripheral blood lymphocytes of astronauts were prepared before and after 120-198 days of stay at the International Space Station (ISS), and CAs were compared in the two lymphocyte samples from each astronaut. It was reported that the frequency of CA significantly increased after their stay in space (Obe et al., 1997). ISS missions have been conducted since 2000 (Feiveson et al., 2021; George et al., 2013), and the length of stay has been steadily increasing from about 3-6 months to over a year; in fact, 
the longest single mission in space is 437.7 days. Moreover, some astronauts participate in multiple ISS missions, such that the longest cumulative stay in space is 2.2 years. It has been reported that astronauts receive about $0.4 \mathrm{mSv} /$ day of radiation on the ISS, which stays in low Earth orbit (Sato et al., 2018). The ISS is located inside the Van Allen Belt; therefore, heavy-ion beams coming from deep space cannot reach it because they interact with protons, alpha rays, and electrons trapped in Earth's magnetic field. In deep space outside of Earth's magnetic field, it is predicted that astronauts will receive about $1 \mathrm{mSv} /$ day during the solar minimum (Sato et al., 2018), or about $1 \mathrm{~Sv}$ during a 2.5-year mission to Mars (Hassler et al., 2014) due to heavy-ion beams with higher LET. The longer the duration of future human space missions such as living on the Moon and Mars, the greater the exposure to space radiation, and the higher the potential health risk to humans will be.

In addition to radiation, the space environment is practically free of gravitational force, or the world of microgravity $(\mu G)$, and living organisms in space are exposed to these two environmental factors at the same time. We have been studying whether simultaneous exposure of cells to simulated $\mu G$ and space radiation is different from exposing them to only one of the two. A three-dimensional (3D) clinostat was used to simulate the $\mu G$ condition by continuously rotating it on two axes. By synchronizing the specimen position on the clinostat with the timing of irradiation, we were able to avoid unnecessary stoppage of rotation during irradiation and achieve simultaneous exposure to simulated $\mu G$ and radiation (Ikeda et al., 2016, 2017). Using this system, we irradiated human fibroblasts while they were exposed to simulated $\mu G$. Cells were first kept under simulated $\mu G$ for 3 or 24 hours and then irradiated with X-ray or carbon-ion (C-ion) beam while still being under simulated $\mu \mathrm{G}$. Control cells were kept on a stationary clinostat for the same lengths of time and irradiated in the same manner. We found that the expression of some cell-cycle inhibitor genes was decreased while that of certain cell-cyclepromoting genes was increased (lkeda et al., 2019). In another study of ours, human fibroblasts irradiated under simulated $\mu G$ conditions showed a higher frequency of both simple and complex types of CA compared with cells irradiated under the $1 G$ condition (Hada et al., 2019). The results from combined effects of simulated $\mu G$ and $C$-ion beam indicates that cells damaged by the radiation are not properly arrested at the cell-cycle checkpoint and may proliferate without repairing the damage. Furthermore, our recent study showed that the frequency of CA was increased in human lymphoblastic cells, which are nonadherent cells, when exposed to simulated $\mu G$ and radiation (Yamanouchi et al., 2020). Our studies have suggested that different cell types are affected differently by the simultaneous exposure to simulated $\mu G$ and radiation, thus making it important to analyze the combined effects on various cell types in the human body. In this study, we investigated the combined effects from the viewpoint of CAs in using human peripheral blood lymphocytes.

\section{Materials and Methods}

\section{Human blood}

This study was conducted in accordance with accepted ethical and humane practices, and approved by the Institutional Review Board (IRB) of Gunma University (IRB no. HS2019-232) and Prairie View A\&M University (IRB no. 2019-079). All experiments were performed using whole peripheral blood drawn from a healthy, nonsmoking volunteer just before the experiment.

\section{Cell cultures simultaneously exposed to simulated $\mu G$ and radiation}

A sterile polypropylene bag was formed and filled completely with $5 \mathrm{~mL}$ of the freshly drawn whole blood (Fig. 1) and fitted into a diamond-shaped cavity of the (a)

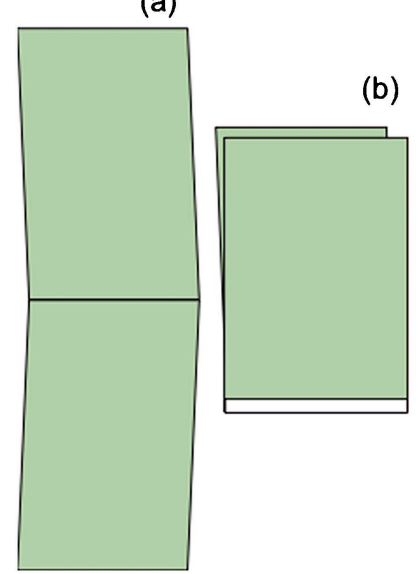

(c)

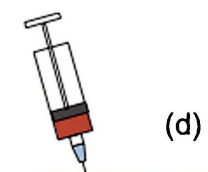

(e)

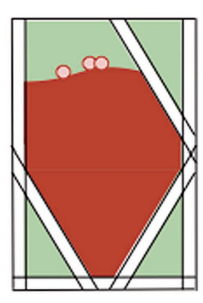

(f)

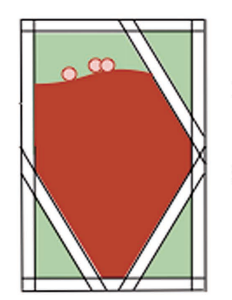

(g)

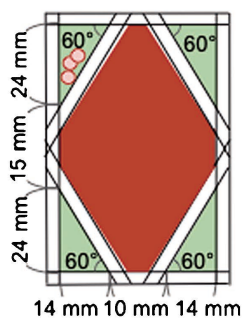

Figure 1. The process of making a sterile blood bag. A sterile polypropylene sheet was folded in half (a), and the folded side was thermo-compressed (b). The two longer sides of the sterile bag were thermo-compressed, and three edges were thermo-compressed to form a diamond shape (c). Blood was carefully injected into the bag with a syringe so as not to puncture it (d). Some air bubbles will form (e). The remaining shorter side of the sterile bag is thermo-compressed (f), and the last edge of the diamond-shaped bag is then thermo-compressed while excluding air bubbles from the blood filled compartment $(\mathrm{g})$. 
sample folder on the clinostat. To make the bag, a polypropylene sheet (Hybrid MekkinBag HM-1304, HOGY, Osaka, Japan) was folded in half (Fig. 1a), and the folded, short side was thermo-compressed (Fig. 1b). The two long sides were then thermos-compressed, followed by thermo-sealing three of the diamond-shaped bag edges (Fig. 1c). The blood was carefully injected into the bag with a syringe (Fig. 1d), during which some air bubbles may form (Fig. 1e). The one remaining side of the sterile bag was then thermo-compressed (Fig. 1f) after all bubbles and air space were chased out from the bag (Fig. 1g).

The bag containing blood was mounted on the 3D clinostat sample stage, in order to have efficient heat transfer from the stage $\left(37^{\circ} \mathrm{C}\right)$ to the blood in the bag. This also ensured stable mounting of the bag onto the clinostat sample stage. The clinostat was turned on so that the cells were acclimatized to simulated $\mu G$ for 6 hours before irradiation. The 3D clinostat rotates on two axes, $11 \mathrm{rpm}(66 \%$ second) in the $X$-axis direction and $13 \mathrm{rpm}\left(78^{\circ} /\right.$ second) in the $\mathrm{Y}$-axis direction. By constantly changing the sample orientation relative to the direction of Earth's gravity, the time-averaged gravity vector acting on cells can be brought to close to zero. Samples were exposed for 0.2 seconds of each 60 -second cycle when the stage becomes perpendicular to the axis of radiation beam. For X-ray irradiation, we used the $\mathrm{X}$-ray generator (200 kV, $14.6 \mathrm{~mA}, 0.3 \mathrm{~mm}$ aluminum filter, MultiRad225: Faxitron Bioptics, LLC, Tucson, AZ, USA) with a high speed shutter (Accelerator Engineering Co., Chiba, Japan) which was synchronized to the clinostat rotation so that the shutter opened only when the sample bag was correctly oriented relative to the beam axis. For synchronized $\mathrm{C}$-ion beam irradiation, a synchrotron
(Gunma University Heavy Ion Medical Center, Gunma, Japan) and the respiratory gating system were used. The dose-averaged LET was $50 \mathrm{keV} / \mu \mathrm{m}$ at the center of the 6 -cm spread-out Bragg distribution with an energy of $290 \mathrm{MeV} / \mathrm{n}$. Simultaneous exposure to simulated $\mu \mathrm{G}$ and radiation was performed without stopping clinostat rotation. For $1 \mathrm{G}$ control, cells were mounted on an appropriately oriented stationary clinostat and irradiated in the same manner as described earlier (Ikeda et al., 2016, 2017; Ohno et al., 2011; Takahashi et al., 2017). The dose rate for both $\mathrm{X}$-ray and $\mathrm{C}$-ion beam was approximately $0.03 \mathrm{~Gy} /$ minute.

\section{Chromosome collection from the whole blood sample}

Figure 2 shows the overall flow of the experiment. The method of chromosome collection was described in our previous reports (Hada et al., 2019; Yamanouchi et al., 2020). Samples were irradiated with 0.5 or $1.0 \mathrm{~Gy}$ of X-ray, or $0.1,0.25$ or 0.5 Gy of C-ion beam 6 hours after gravitational treatment started and kept under $1 G$ or simulated $\mu G$ condition for 24 hours to allow DNA damage to repair. The whole blood was then transferred into T25 flask with PB-max karyotyping medium and cultured at $37^{\circ} \mathrm{C}$ for 52 hours. Chromosomes in $\mathrm{G} 2$ and mitotic stages in the first cell division were collected using the chemically induced premature chromosome condensation (PCC) method described previously (Durante et al., 1999; George et al., 2015). 50 nM Calyculin A (FUJIFILM Wako Pure Chemical Co., Osaka, Japan) was added to the culture 30 minutes prior to harvesting, and cells were treated with a hypotonic solution $(0.075 \mathrm{M} \mathrm{KCl})$ for 15 minutes at $37^{\circ} \mathrm{C}$ and fixed with methanol/acetic acid (3:1).

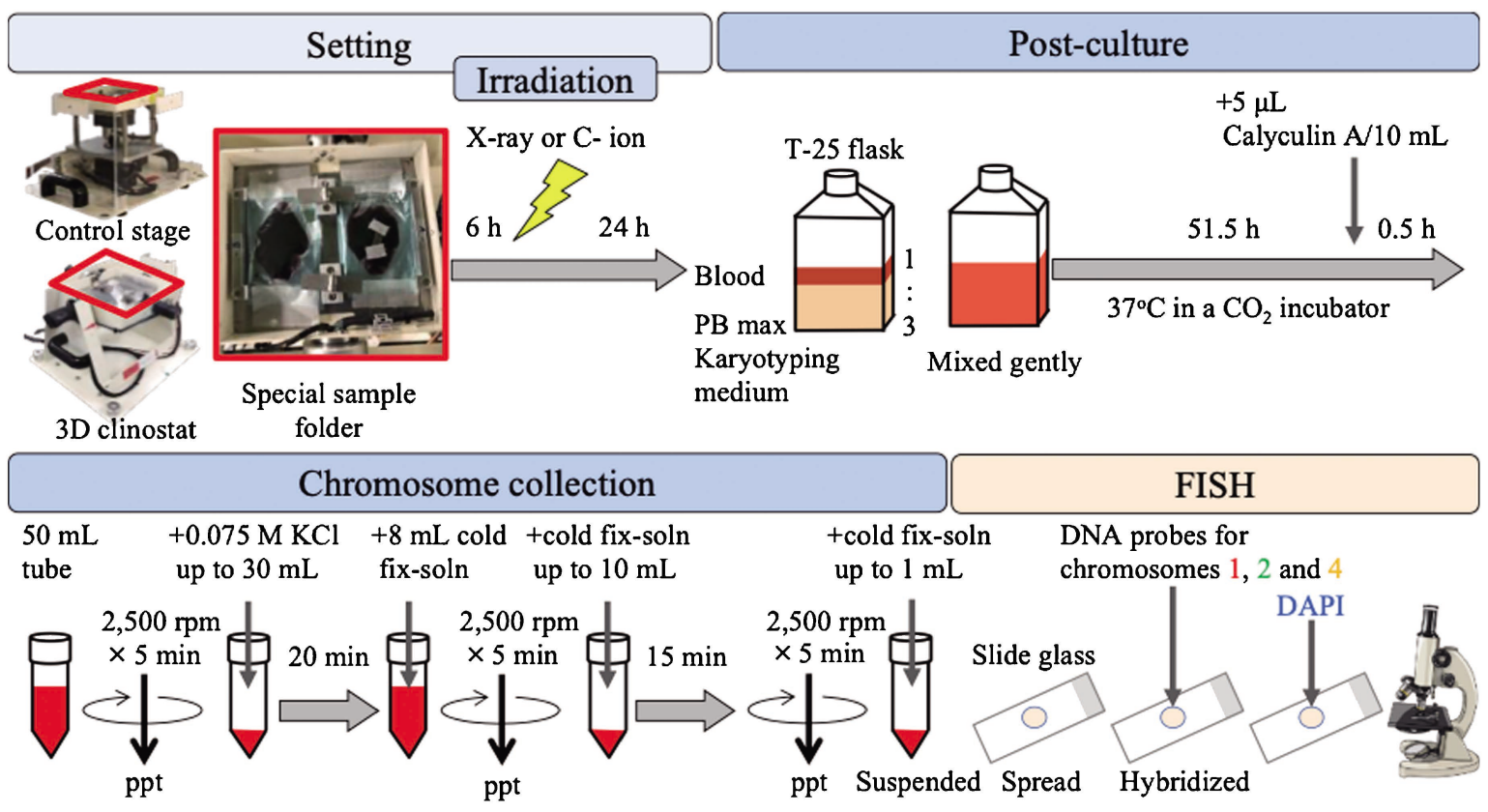

Figure 2. The overall flow of experiment. 


\section{$\mathrm{FISH}$}

The FISH method was described in our previous reports (Hada et al., 2019; Yamanouchi et al., 2020). Fixed cells were dropped on a slide and hybridized in situ with the three-color fluorescence whole-chromosome human DNA probe (Aquarius, Cytocell, Oxford Gene Technology, Oxfordshire, UK) to stain chromosomes 1 to red, 2 to green, and 4 to yellow, according to the protocol recommended by the manufacturer. After staining these target chromosomes, all others were stained with 4,6-diamidino-2-phenylindole (DAPI; blue). When breaks occurred in any two chromosomes (resulting in dicentrics or translocations due to the exchange), this was defined as a simple type of CA (George et al., 2009). Incomplete translocation and incomplete dicentrics were also classified as a simple type of CA, assuming that the mutual fragments were below the detection level. A complex type of CA was defined as the occurrence of three or more breaks and exchanges in two or more chromosomes; each type of CA was counted as one exchange. When more than two chromosomes were damaged in a single cell, they were separately evaluated. We aimed to analyze at least 1,000 cells or to detect 50 aberrant cells in each type of radiation and each dose.

\section{Statistical analysis}

Statistical analysis was performed as previously described (Hada et al., 2019; Yamanouchi et al., 2020), and the ratio between the scored and the total cells analyzed was defined as the frequency of CA. It has been reported that radiation damage randomly occurs among chromosomes and that CA is proportional to the DNA content of the chromosome analyzed, with some variability in the data (Luomahaara et al., 1999). The following formula was used to convert the frequency of $\mathrm{CA}$ of chromosomes 1, 2, and 4 to whole-genome equivalents (Lucas et al., 1992):

$F p=2.05[f p(1-f p)+f p 1 f p 2+f p 1 f p 3+f p 2 f p 3] F G$, where $f p$ is the fraction of the whole genome comprised of painted chromosomes; fp1, fp2, and fp3 are the fractions of the genome for chromosomes 1,2, and 4, respectively; and FG is the whole-genome aberration frequency. $\mathrm{Fp}$ is the combined frequency of exchanges in all painted chromosomes.

Standard errors for aberration frequencies were calculated assuming Poisson statistics. The error bars in the figure represent the standard errors of the mean values. The Fisher's exact test was used to assess whether the difference in frequency of chromosome exchanges is significant between different treatment groups. Logistic regression analysis was performed to examine the effect of radiation dose and gravity on total exchanges, using total exchange as dependent variable and radiation dose and gravity as independent variables.

\section{Results}

CAs in human peripheral blood lymphocytes simultaneously exposed to simulated $\mu G$ and radiation

Table 1 shows the whole-genome equivalent frequency of CAs per 100 cells in human peripheral blood lymphocytes irradiated by $\mathrm{X}$-ray or $\mathrm{C}$-ion beam under the $1 G$ or simulated $\mu G$ conditions. The $p$-values were calculated by the Fisher's exact test between the $1 G$ and the simulated $\mu G$ conditions at each dose. Values less than 0.05 are shown in bold. Six cases were found to be $p<0.05$ : the frequencies of simple and total CAs in cells

Table 1. Whole-genome equivalent for the frequency of CAs per 100 human peripheral blood lymphocytes irradiated by X-ray or C-ion beam under $1 G$ or simulated $\mu G$ condition. $P$-values were obtained from the Fisher's exact test between $1 G$ and simulated $\mu G$ conditions at each dose. $P<0.05$ values are shown in bold.

\begin{tabular}{|c|c|c|c|c|c|c|c|c|}
\hline Radiation & Gravity & $\begin{array}{l}\text { Total spread } \\
\text { scored }\end{array}$ & $\begin{array}{c}\text { No. of aberrant } \\
\text { spreads }\end{array}$ & $\begin{array}{c}\text { Simple } \\
\text { exchanges }\end{array}$ & $\%$ & $\begin{array}{c}\text { Complex } \\
\text { exchanges }\end{array}$ & $\%$ & $\begin{array}{c}\text { Total } \\
\text { exchanges }\end{array}$ \\
\hline \multirow{3}{*}{$\begin{array}{l}\text { Control } \\
\text { (0 Gy) }\end{array}$} & $1 G$ & 3018 & 18 & $0.77 \pm 0.26$ & 100.00 & 0 & 0.00 & $0.77 \pm 0.26$ \\
\hline & $\mu G$ & 1008 & 6 & $1.02 \pm 0.51$ & 100.00 & 0 & 0.00 & $1.02 \pm 0.51$ \\
\hline & & ( $p$-value) & & 0.748 & & 1 & & 0.748 \\
\hline \multirow{3}{*}{$\begin{array}{l}\text { X-ray } \\
\text { (0.5 Gy) }\end{array}$} & $1 G$ & 1037 & 37 & $7.93 \pm 1.40$ & 91.46 & $0.74 \pm 0.43$ & 8.54 & $8.67 \pm 1.47$ \\
\hline & $\mu G$ & 782 & 50 & $12.82 \pm 2.05$ & 82.98 & $2.63 \pm 0.93$ & 17.02 & $15.45 \pm 2.25$ \\
\hline & & ( $p$-value) & & 0.0497 & & 0.065 & & 0.0085 \\
\hline \multirow{3}{*}{$\begin{array}{l}\text { X-ray } \\
(1.0 \mathrm{~Gy})\end{array}$} & $1 G$ & 685 & 50 & $15.38 \pm 2.40$ & 93.16 & $1.13 \pm 0.65$ & 6.84 & $16.51 \pm 2.49$ \\
\hline & $\mu G$ & 419 & 50 & $32.51 \pm 4.47$ & 96.35 & $1.23 \pm 0.87$ & 3.65 & $33.74 \pm 4.55$ \\
\hline & & ( $p$-value) & & 0.000211 & & 1 & & 0.00021 \\
\hline C-ion & $1 G$ & 1018 & 18 & $2.78 \pm 0.84$ & 78.75 & $0.76 \pm 0.44$ & 21.53 & $3.53 \pm 0.94$ \\
\hline beam & $\mu G$ & 1025 & 25 & $5.01 \pm 1.12$ & 86.83 & $0.75 \pm 0.43$ & 13.00 & $5.77 \pm 1.20$ \\
\hline (0.1 Gy) & & ( $p$-value) & & 0.147 & & 1 & & 0.184 \\
\hline C-ion & $1 G$ & 1050 & 50 & $9.79 \pm 1.55$ & 76.90 & $2.94 \pm 0.85$ & 23.10 & $12.73 \pm 1.77$ \\
\hline beam & $\mu G$ & 894 & 50 & $13.51 \pm 1.97$ & 81.04 & $3.16 \pm 0.95$ & 18.96 & $16.67 \pm 2.19$ \\
\hline (0.25 Gy) & & ( $p$-value) & & 0.152 & & 1 & & 0.168 \\
\hline C-ion & $1 G$ & 403 & 50 & $24.23 \pm 3.93$ & 65.50 & $12.75 \pm 2.85$ & 34.47 & $36.99 \pm 4.86$ \\
\hline beam & $\mu G$ & 264 & 48 & $45.75 \pm 6.67$ & 77.05 & $13.63 \pm 3.64$ & 22.95 & $59.38 \pm 7.60$ \\
\hline (0.5 Gy) & & ( $p$-value) & & 0.0019 & & 0.859 & & 0.0051 \\
\hline
\end{tabular}

${ }^{*}$ The results are presented as mean \pm standard error of the mean. 
Sakuya Yamanouchi et al.

Table 2. Logistic-regression analysis of the effect of radiation dose and gravity on total exchanges.

\begin{tabular}{|c|c|c|c|c|c|c|c|}
\hline \multirow{2}{*}{ Radiation } & \multirow{2}{*}{ Factor } & \multirow{2}{*}{ Coefficient } & \multirow{2}{*}{$\begin{array}{c}\mathrm{SE}^{*} \text { of } \\
\text { coefficient }\end{array}$} & \multirow{2}{*}{$p$-value } & \multirow{2}{*}{$\mathrm{OR}^{\star *}$} & \multicolumn{2}{|c|}{$95 \% \mathrm{Cl}^{\star * \star}$ for OR } \\
\hline & & & & & & Lower & Lower \\
\hline \multirow{3}{*}{ X-ray } & Dose & 2.732 & 0.203 & $3.65 \times 10^{-41}$ & 15.370 & 10.318 & 22.895 \\
\hline & Gravity & 0.760 & 0.150 & $4.00 \times 10^{-7}$ & 2.138 & 1.594 & 2.868 \\
\hline & Constant & -5.247 & 0.178 & $1.85 \times 10^{-191}$ & & & \\
\hline \multirow{3}{*}{ C-ion beam } & Dose & 6.284 & 0.355 & $3.13 \times 10^{-70}$ & 535.886 & 267.397 & 1073.961 \\
\hline & Gravity & 0.466 & 0.124 & $1.66 \times 10^{-4}$ & 1.594 & 1.250 & 2.031 \\
\hline & Constant & -5.490 & 0.146 & $<1.00 \times 10^{-191}$ & & & \\
\hline
\end{tabular}

${ }^{\star} \mathrm{SE}$, standard error; ${ }^{* *} \mathrm{OR}$, odds ratio; ${ }^{* *} \mathrm{Cl}$, confidence interval.

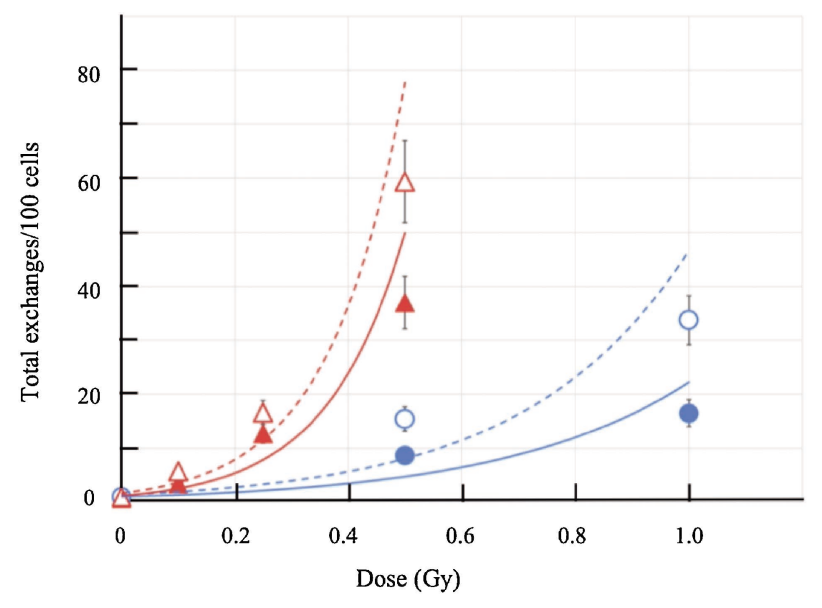

Figure 3. Frequencies of total chromosome exchanges induced by X-ray $(\bullet \circ)$ or $\mathrm{C}$-ion beam $(\boldsymbol{\Delta} \triangle)$ while cells were under either $1 G(\bullet \Delta)$ or simulated $\mu G$ condition $(\bigcirc \Delta)$. Error bars indicate standard error of the mean. The " $n$ " value for each data point is shown in Table 1. The dotted and solid lines show the results of linear approximation.

irradiated with 0.5 or 1.0 Gy X-ray, or 0.5 Gy C-ion beam. Looking at the total exchange, simulated $\mu G$ per se does not appear to cause a significant change in $C A$ in peripheral blood lymphocytes, but both X-ray and C-ion beam at the dose higher than $0.5 \mathrm{~Gy}$ induce CAs under the simulated $\mu G$ condition. Note that low doses $(0.1$ and $0.25 \mathrm{~Gy}$ ) of $\mathrm{C}$-ion beam irradiation did not show increased CAs under simulated $\mu G$.

Table 2 shows the results of the logistic-regression analysis of the effect of radiation dose or gravity on the frequency of total CAs. For the odds ratio for X-ray, the dose was 15.370 and the gravity was 2.138 . The odds ratios for C-ion beam were 535.886 and 1.594 for the dose and the gravity, respectively. These results indicate that CAs were increased 2.1 times by X-ray and 1.6 times by $\mathrm{C}$-ion beam in the cells irradiated under simulated $\mu G$ compared with $1 G$, regardless of irradiation doses. These results also show that $\mathrm{C}$-ion beam causes many more CAs than X-ray.

Figure 3 shows the relationship between irradiation doses and total exchanges. The frequency of CAs increased in a dose-dependent manner for both X-ray (blue) and C-ion beam (red). The frequency of CAs was
Table 3. Ratio of frequencies of CAs in cells irradiated with $\mathrm{C}$-ion beam to cells irradiated with X-rays at the same 0.5 Gy dose.

\begin{tabular}{|c|c|c|c|c|}
\hline \multirow{2}{*}{$\begin{array}{l}\text { Type of } \\
\text { CAs }\end{array}$} & \multirow{2}{*}{ Gravity } & \multicolumn{2}{|c|}{ Radiation } & \multirow{2}{*}{ Ratio } \\
\hline & & X-ray & C-ion beam & \\
\hline \multirow{2}{*}{ Total } & $1 G$ & 8.67 & 36.99 & 4.27 \\
\hline & $\mu G$ & 15.45 & 59.38 & 3.84 \\
\hline \multirow{2}{*}{ Single } & $1 G$ & 7.93 & 24.23 & 3.06 \\
\hline & $\mu G$ & 12.82 & 47.75 & 3.72 \\
\hline \multirow{2}{*}{ Complex } & $1 G$ & 0.74 & 12.75 & 17.22 \\
\hline & $\mu G$ & 2.63 & 13.63 & 5.18 \\
\hline
\end{tabular}

higher in cells irradiated under simulated $\mu G$ compared with those irradiated at $1 G$ for both types of irradiating beams. The graph also shows that C-beam is more hazardous to lymphocytes than X-ray. Table 3 shows the ratio of frequencies of CAs in the cell irradiated with $\mathrm{C}$-ion beam to the cell irradiated with X-rays at the same $0.5 \mathrm{~Gy}$ dose. The ratios were calculated by dividing the frequency of CAs in the cells irradiated by $\mathrm{C}$-ion beam with that in the cells irradiated with X-ray. The frequency of CAs increased 4.3 times at $1 G$ and 3.8 times at simulated $\mu G$ in the cells irradiated with $\mathrm{C}$-ion beam than in the cells irradiated with X-ray.

Figure 4 shows a more detailed analysis on the occurrence of simple (blue bar) and complex (red bar) types of CAs under various conditions. No complex type was detected in unirradiated control cells. Compared with $\mathrm{C}$-ion beam irradiated cells, the frequency of CAs was lower in X-ray irradiated cells. In Table 3, at the same dose of $0.5 \mathrm{~Gy}$, the frequency of the simple type of CAs increased 3.1 times at $1 G$ and 3.7 times at simulated $\mu G$. The frequency of complex type increased 17 times at $1 G$ and 5.2 times at simulated $\mu G$. These ratio data show that $\mathrm{C}$-ion beam induces more CAs than $\mathrm{X}$-ray.

\section{Discussion}

\section{Advantage of using human peripheral blood lymphocytes}

Many studies have found increased levels of CAs in astronauts' blood lymphocytes after space missions. For example, it was reported that increased frequencies of CAs were detected in blood lymphocytes of eight astronauts who returned from separate long space missions (George et al., 2001). Another study reported 


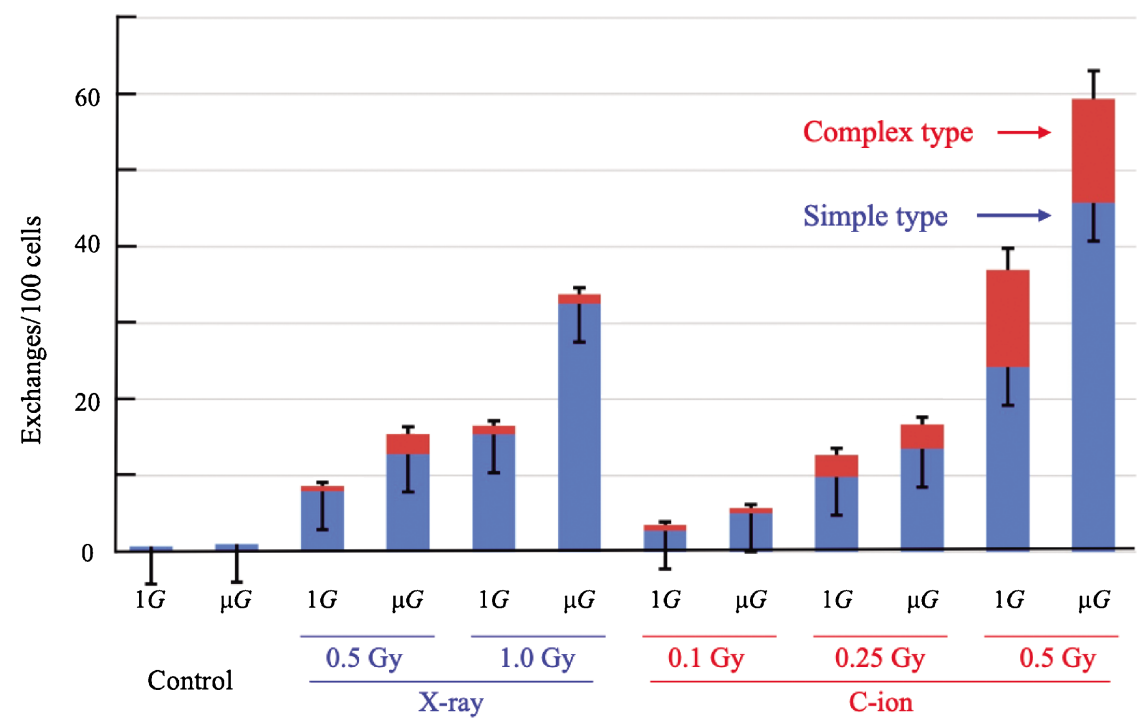

Figure 4. Frequencies of simple and complex types of CAs induced by $\mathrm{X}$-ray or $\mathrm{C}$-ion beam while cells are under either $1 G$ or simulated $\mu G$ condition. The blue column shows the simple type of CAs, and the red column shows the complex type. Error bars indicate standard error of the mean. "n" is shown in Table 1.

that lymphocytes of astronauts who were in a 115 dayspace mission exhibited a significantly higher number of CAs compared with abnormalities scored in preflight samples from the same individuals (Yang et al., 1997). Additionally, when the frequency of CAs was measured in the peripheral blood lymphocytes of 33 astronauts who participated in space missions over a period of 11 years, a statistically significant increase was found in astronauts from long-term missions (Durante et al., 2003). However, there were no changes in the $\mathrm{CA}$ frequencies of two astronauts who experienced only a nine-day spaceshuttle mission (Wu et al., 2001), and no significant differences were observed after short-term taxi flights (Durante et al., 2003). In another study, when CA frequencies were determined in 9 individuals who spent varying lengths of time in space, no correlation was found between $\mathrm{CA}$ and the number of flights or the duration of the astronaut's stay in space (Greco et al., 2003). Despite a short exposure to simulated $\mu G$ for only 54 hours, this in vitro study shows an increase in the frequency of CAs in human peripheral blood lymphocytes under simulated $\mu G$ compared with $1 G$ (Fig. 3). Under a human research program by NASA, preflight blood samples from astronauts were exposed ex vivo to varying doses of gamma rays, and the dose-response for CA rate and the background (i.e. base line) aberration have been determined for each astronaut. It was found that the CA rate observed in the crewmembers can be fairly well predicted by the radiosensitivity determined by the preflight ex vivo experiments after adjusting for the actual dose received during the mission and the base line CA levels (Feiveson et al. 2021). Since peripheral blood lymphocytes are used as biodosimetry for risk assessment of astronauts, there is an advantage to use these cells in ground based experiments in order to obtain and predict biological effects of the space environment. Such data will complement the limited availability of data from astronauts.

\section{The difference between $X$-ray and $C$-ion beam irradiation}

The frequency of the complex type of CAs increased in cells irradiated by $\mathrm{C}$-ion beam compared with those irradiated by X-ray with the same dose (Fig. 4). It has been reported that blood lymphocytes of astronauts after prolonged space flights show a slightly higher frequency of complex CAs perhaps due to exposure to high-LET radiation (George et al., 2001). DSB is the most dangerous type of DNA damage (Shibata and Jeggo, 2014), and LET is defined as the amount of energy given to a tissue by ionizing radiation per unit length (Danzker et al., 1959). X-rays are a form of low-LET radiation and mostly produce single-strand breaks (SSBs), with only a smaller number of DSBs. On the other hand, high-LET heavy-ion beams in space produce dense DSBs due to their high-energy density (Löbrich et al., 1996) and have a high biological lethality. The relative biological effects of C-ion beam compared with X-ray is 2-3 times higher for human lymphoblastic TK6 cells (Yamanouchi et al., 2020). It has also been suggested that heavy-ion irradiation induces complex DNA damages, called a clustered DNA damage, which is defined as the damage that includes DSBs and SSBs within one or two helical turns of DNA (Hada et al., 2008; Pinto et al., 2005; Rydberg et al., 1998). These clustered damages have been reported to be either irreparable or likely to result in misrepair due to delayed repair (Pinto et al., 2005; Shibata and Jeggo, 2014). Although the mechanism for the increased CA formation by high-LET radiation is not fully understand, clustered formation of DNA damage may play a role in inducing a higher frequency of the complex type of CAs 
by $\mathrm{C}$-ion beam irradiation.

The total exchanges in human peripheral blood lymphocytes compared with human lymphoblastic TK6 cells

We have earlier reported combined effects of radiation and simulated $\mu G$ on TK6 cells which are stable human lymphoblastic cell line (Yamanouchi et al., 2020). Suppl. 1 shows the fold increase in total number of CAs in human blood lymphocytes compared to TK6 cells. By comparing the total number of CAs in 100 human blood lymphocytes with that in 100 TK6 cells, we found that the CA frequency was lower in human lymphocytes than in TK6 cells at the baseline (i.e. without irradiation at $1 G$ ). However, when cells were irradiated, more CAs were observed in lymphocytes obtained from the peripheral blood of healthy humans than in TK6 cells with both X-ray and C-ion beam irradiation. At all doses and in both cell types, more CAs were observed at simulated $\mu G$ than at $1 G$. Although the number of $C A s$ in the control lymphocyte samples was less than that of TK6 cells, human blood lymphocytes are considered to be radiosensitive because they produce more CAs upon irradiation. Cell survival data are also needed to evaluate more accurately the radiation/gravity effects on different cell types.

The total exchanges in human peripheral blood lymphocytes compared with human fibroblasts $1 B R$ hTERT cells

We have also reported earlier on the effects of simultaneous exposure of human fibroblasts 1BR-hTERT cells to simulated $\mu G$ and radiation (Hada et al., 2019). Suppl. 1 shows the data from this earlier study. The comparison of data from the lymphocytes and 1BRhTERT cells indicate that under the same irradiation doses, more CA was observed in human peripheral blood lymphocytes than in 1BR-hTERT cells with both X-ray and $\mathrm{C}$-ion beam irradiation. At both doses and in both cell types, more CAs were observed at simulated $\mu G$ than at $1 G$, and the rate of increase in CAs was not higher at simulated $\mu G$.

Suppl. 1. Total number of CAs in peripheral blood lymphocytes, TK6 cells and 1BR-hTERT cells irradiated by X-ray or C-ion beam under $1 G$ or simulated $\mu G$ condition.

\begin{tabular}{ccrrr}
\hline & & \multicolumn{3}{c}{ Total number of CAs } \\
\cline { 3 - 5 } Radiation & Gravity & $\begin{array}{c}\text { Human } \\
\text { blood } \\
\text { lymphocytes }\end{array}$ & TK6 cells* & $\begin{array}{c}\text { 1BR-hTERT } \\
\text { cells }\end{array}$ \\
\hline Control & $1 G$ & $0.77 \pm 0.26$ & $2.36 \pm 0.75$ & $0.73 \pm 0.30$ \\
(0 Gy) & $\mu G$ & $1.02 \pm 0.51$ & $3.14 \pm 0.87$ & $1.37 \pm 0.41$ \\
\hline X-ray & $1 G$ & $8.67 \pm 1.47$ & $5.03 \pm 1.10$ & $3.39 \pm 0.91$ \\
$(0.5$ Gy) & $\mu G$ & $15.45 \pm 2.25$ & $8.59 \pm 1.43$ & $5.58 \pm 1.00$ \\
\hline C-ion beam & $1 G$ & $12.73 \pm 1.77$ & $6.71 \pm 1.27$ & \\
(0.25 Gy) & $\mu G$ & $16.67 \pm 2.19$ & $10.31 \pm 1.77$ & \\
\hline C-ion beam & $1 G$ & $36.99 \pm 4.86$ & $25.63 \pm 3.82$ & $15.42 \pm 2.44$ \\
$(0.5$ Gy) & $\mu G$ & $59.38 \pm 7.60$ & $36.02 \pm 5.09$ & $18.83 \pm 2.87$ \\
\hline
\end{tabular}

*Yamanouchi et al., 2020, **Hada et al., 2019.
The combined effect of simulated $\mu G$ and radiation

Five out of 10 comparisons of combined effects yielded statistically significant differences $(p<0.05)$ between $1 G$ and simulated $\mu G$ (Table 1). Logisticregression analysis was performed to investigate the effect of simulated $\mu G$ on CA frequency in detail, and it was found that simulated $\mu G$ made a significant contribution (Table 2). Compared with the $1 G$ condition, 2.1 times more CAs were produced in cells irradiated with $\mathrm{X}$-ray and 1.6 times more were produced in cells irradiated with C-ion beam under simulated $\mu G$, regardless of the dose. Although the odds ratios for dose are as large as 15.370 for X-ray and 535.886 for $\mathrm{C}$-ion beam, the dose contribution is expected to be very small because the space-radiation dose is small.

\section{Conclusions, limitations and future plans}

Studies on astronauts' blood lymphocytes have repeatedly suggested that long-term space missions increase CAs, presumably caused by both space radiation and $\mu G$. Although they provide precious information, these studies are severely limited by a small case number. Also, quality and quantity of the radiation that astronauts are exposed during ISS missions are different from the expected radiation in the future deep space missions. For these reasons, more ground-based experimental model systems need to be developed. The system we used for the current study has been described in detail (Ikeda et al., 2016, 2017) and may be one such model system because cells can be exposed simultaneously to simulated $\mu G$ and radiation, albeit for a shorter period of time, and the results obtained using this system are remarkably similar to many of the findings obtained using astronauts' peripheral blood lymphocytes. Our results demonstrate the usefulness of this system for predicting certain effects of the space environment on human cells in space. Moreover, this study demonstrates that the system is amenable for testing not only adherent cells but also floating cells, and perhaps ex vivo tissue pieces. Using the current system, we are planning to expand the current study by collecting more samples from healthy donors and to determine whether disparities exist among individuals, ages and sex.

However, this study has a few limitations. First, the experiment was conducted at the cellular level, and applying such in vitro results to organismic physiology is challenging. Second, the experiments performed on the ground do not fully recapitulate biological events that takes place in the actual space environment. The Artemis Project proposed by NASA aims for human beings to inhabit on the Moon and Mars. The gravitational forces to which astronauts will be exposed in these projects are $1 / 6 G$ on the Moon and $3 / 8 G$ on Mars as well as $\mu G$ in space while traveling. Thus, it is important to study also the effects of partial gravity. To ensure safe and low-risk living in space, it is critical to elucidate molecular mechanisms for biological effects of the space environment. Such information is necessary for developing ways to mitigate negative effects of the space environment on life. In addition to simulated $\mu G$, we have 
recently developed devices for ground experiments that simulate various partial gravity (Takahashi et al., 2020). In the future work, we plan to compare our chromosomal aberration findings with simulated $\mu G$ to partial gravity conditions using these devices. It is also important to investigate if and how the biological system, at both the cellular and organismic levels, is affected by rapid changes in gravitational environments such as departure from Earth or returning to Earth. Studies on the effects of changing gravity from $1 G$ to simulated $\mu G$ on gene expressions and protein modifications are in progress using our devices.

\section{Acknowledgments}

This work was supported by NASA Space Biology Program (grant no. 80NSSC19K0133) (M.H., K.F., and A.T.), JAXA Front Loading Study (A.T., Y.Y., and M.H.), Gunma University for the Promotion of Scientific Research (A.T.), Gunma University Graduate School of Medicine for the Activation of Research Activity (S.Y.), and TAMU Chancellor's Research Initiative, Texas A\&M University (P.B.S.). This work was the result of using research equipment shared in MEXT Project for Promoting Public Utilization of Advanced Research Infrastructure (Program for supporting introduction of the new sharing system) (grant no. JPMXS0430300120) (S.Y. and A.T.) and Research Projects (20B-4) with Heavy lons at the Gunma University Heavy Ion Medical Center (GHMC) (S.Y., Y.Y., and A.T.).

We thank the member of Accelerator Engineering Co. for supporting $\mathrm{C}$-ion beam irradiation.

\section{Conflicts of Interest}

The authors declare no competing interests.

\section{References}

Anderson, R. M., Marsden, S. J., Wright, E. G., Kadhim, M. A., Goodhead, D. T. and Griffin, C. S. (2000) Complex chromosome aberrations in peripheral blood lymphocytes as a potential biomarker of exposure to high-LET alpha-particles. Int. J. Radiat. Biol., 76, 31-42.

Danzker, M., Kessaris, N. D. and Laughlin, J. S. (1959) Absorbed dose and linear energy transfer in radiation experiments. Radiology, 72, 51-61.

Durante, M., Furusawa, Y., Majima, H., Kawata, T. and Gotoh, E. (1999) Association between G2-phase block and repair of radiation-induced chromosome fragments in human lymphocytes. Radiat. Res., 151, 670-676.

Durante, M., Snigiryova, G., Akaeva, E., Bogomazova, A., Druzhinin, S., Fedorenko, B., Greco, O., Novitskaya, N., Rubanovich, A., Shevchenko, V., et al. (2003) Chromosome aberration dosimetry in cosmonauts after single or multiple space flights. Cytogenet. Genome Res., 103, 40-46.

Edwards, A. A. and Lloyd, D. C. (1991) Chromosomal damage in human lymphocytes: Effect of radiation quality. In: Fielden E. M., O'Neill P. (eds) The Early Effects of Radiation on DNA. NATO ASI Series (Series $\mathrm{H}$ : Cell Biology), 54. Springer, Berlin, Heidelberg.
Feiveson, A., George, K., Shavers, M., Moreno Villanueva, M., Zhang, Y., Babiak Vazquez, A., Crucian, B., Semones, E. and Wu, H. (2021) Predicting chromosome damage in astronauts participating in international space station missions. Sci. Rep., 11, 5293.

Ferguson-Smith, M. A. (2015) History and evolution of cytogenetics. Mol. Cytogenet., 8, 19.

Furukawa, S., Nagamatsu, A., Nenoi, M., Fujimori, A., Kakinuma, S., Katsube, T., Wang, B., Tsuruoka, C., Shirai, T., Nakamura, A. J., et al. (2020) Space radiation biology for "Living in Space". BioMed Res. Int., 2020, 4703286.

George, K., Durante, M., Wu, H., Willingham, V., Badhwar, G. and Cucinotta, F. A. (2001) Chromosome aberrations in the blood lymphocytes of astronauts after space flight. Radiat. Res., 156, 731-738.

George, K. A., Hada, M., Jackson, L. J., Elliott, T., Kawata, T., Pluth, J. M. and Cucinotta, F. A. (2009) Dose response of gamma rays and iron nuclei for induction of chromosomal aberrations in normal and repairdeficient cell lines. Radiat. Res., 171, 752-763.

George, K., Rhone, J., Beitman, A. and Cucinotta, F. A. (2013) Cytogenetic damage in the blood lymphocytes of astronauts: Effects of repeat long-duration space missions. Mutat. Res., 756, 165-169.

George, K., Hada, M. and Cucinotta, F. A. (2015) Biological effectiveness of accelerated protons for chromosome exchanges. Front. Oncol., 5, 226.

Greco, O., Durante, M., Gialanella, G., Grossi, G., Pugliese, M., Scampoli, P., Snigiryova, G. and Obe, G. (2003) Biological dosimetry in Russian and Italian astronauts. Adv. Space Res., 31, 1495-1503.

Griffin, C. S., Marsden, S. J., Stevens, D. L., Simpson, P. and Savage, J. R. (1995) Frequencies of complex chromosome exchange aberrations induced by ${ }^{238} \mathrm{Pu}$ alpha-particles and detected by fluorescence in situ hybridization using single chromosome-specific probes. Int. J. Radiat. Biol., 67, 431-439.

Hada, M. and Georgakilas, A. G. (2008) Formation of clustered DNA damage after high-LET irradiation: A review. J. Radiat. Res., 49, 203-210.

Hada, M., Ikeda, H., Rhone, J. R., Beitman, A. J., Plante, I., Souda, H., Yoshida, Y., Held, K. D., Fujiwara, K., Saganti, P. B., et al. (2019) Increased chromosome aberrations in cells exposed simultaneously to simulated microgravity and radiation. Int. J. Mol. Sci., 20, 43.

Hassler, D. M., Zeitlin, C., Wimmer-Schweingruber, R. F., Ehresmann, B., Rafkin, S., Eigenbrode, J. L., Brinza, D. E., Weigle, G., Böttcher, S., Böhm, E., et al. (2014) Mars' surface radiation environment measured with the Mars Science Laboratory's Curiosity rover. Science, 343, 1244797.

Ikeda, H., Souda, H., Puspitasari, A., Held, K. D., Hidema, J., Nikawa, T., Yoshida, Y., Kanai, T. and Takahashi, A. (2016) A new system for three-dimensional clinostat synchronized $\mathrm{X}$-irradiation with a high-speed shutter 
for space radiation research. Biol. Sci. Space, 30, 8-16.

Ikeda, H., Souda, H., Puspitasari, A., Held, K. D., Hidema, J., Nikawa, T., Yoshida, Y., Kanai, T. and Takahashi, A. (2017) Development and performance evaluation of a three-dimensional clinostat synchronized heavy-ion irradiation system. Life Sci. Space Res. (Amst), 12, 5160.

Ikeda, H., Muratani, M., Hidema, J., Hada, M., Fujiwara, K., Souda, H., Yoshida, Y. and Takahashi, A. (2019) Expression profile of cell cycle-related genes in human fibroblasts exposed simultaneously to radiation and simulated microgravity. Int. J. Mol. Sci., 20, 4791.

$\mathrm{Li}, \mathrm{Q}$. (2007) Biomedical research with heavy ions at the IMP accelerators. Adv. Space Res., 40, 455-460.

Lichter, P., Tang, C. C., Call, K., Hermanson, G., Evans, G. A., Housman, D. and Ward, D. C. (1990) High resolution mapping of human chromosome 11 by in situ hybridisation with cosmid clones. Science, 47, 6469.

Loucas, B. D. and Cornforth, M. N. (2001) Complex chromosome exchanges induced by gamma rays in human lymphocytes: An mFISH study. Radiat. Res., 155, 660-671.

Lucas, J. N., Poggensee, M. and Straume, T. (1992) The persistence of chromosome translocations in a radiation worker accidentally exposed to tritium. Cytogenet. Cell Genet., 60, 3-4.

Löbrich, M., Cooper, P. K. and Rydberg, B. (1996) Nonrandom distribution of DNA double-strand breaks induced by particle irradiation. Int. J. Radiat. Biol., 70, 493-503.

Luomahaara, S., Lindholm, C., Mustonen, R. and Salomaa, S. (1999) Distribution of radiation-induced exchange aberrations in human chromosomes 1, 2 and 4. Int. J. Radiat. Biol., 75, 1551-1556.

National Aeronautics and Space Administration. "NASA's Lunar Exploration Program Overview". https://www. nasa.gov/specials/artemis/ (2021/03/14)

Obe, G., Johannes, I., Johannes, C., Hallman, K., Reitz, G. and Facius, R. (1997) Chromosomal aberrations in blood lymphocytes of astronauts after long-term space flights. Int. J. Radiat. Biol., 72, 727-734.

Ohno, T., Kanai, T., Yamada, S., Yusa, K., Tashiro, M., Shimada, H., Torikai, K., Yoshida, Y., Kitada, Y., Katoh, $\mathrm{H}$., et al. (2011) Carbon ion radiotherapy at the Gunma University Heavy Ion Medical Center: New facility setup. Cancers, 3, 4046-4060.

Pinkel, D., Straume, T. and Gray, J. W. (1986) Cytogenetic analysis using quantitative, high-sensitivity, fluorescence hybridization. Proc. Natl. Acad. Sci. U. S. A., 83, 2934-2938.

Pinto, M., Prise, K. M. and Michael, B. D. (2005) Evidence for complexity at the nanometer scale of radiation- induced DNA DSBs as a determinant of rejoining kinetics. Radiat. Res., 164, 73-85.

Rydberg, B., Lobrich, M. and Cooper, P. K. (1998) Repair of clustered DNA damage caused by high LET radiation in human fibroblasts. Phys. Med., 14, 24-28.

Sabatier, L., Al Achkar, W., Hoffschir, F., Luccioni, C. and Dutrillaux, B. (1987) Qualitative study of chromosomal lesions induced by neutrons and neon ions in human lymphocytes at G0 phase. Mutat. Res., 178, 91-97.

Sato, T., Nagamatsu, A., Ueno, H., Kataoka, R., Miyake, S., Takeda, K. and Niita, K. (2018) Comparison of cosmic-ray environments on earth, moon, mars and in spacecraft using PHITS. Radiat. Prot. Dosimetry, 180, 146-149.

Schröck, E., du Manoir, S., Veldman, T., Schoell, B., Wienberg, J., Ferguson-Smith, M. A., Ning, Y., Ledbetter, D. H., Bar-Am, I., Soenksen, D., et al. (1996) Multicolor spectral karyotyping of human chromosomes. Science, 273, 494-497.

Shibata, A. and Jeggo, P. A. (2014) DNA double-strand break repair in a cellular context. Clin. Oncol. (R. Coll. Radiol.), 26, 243-249.

Takahashi, A., Ikeda, H., Souda, H., Puspitasari, A., Held, K. D., Hidema, J., Nikawa, T., Yoshida, Y. and Kanai, T. (2017) Performance evaluation of new devices to synchronize heavy-ion or X-ray irradiation and simulated microgravity for space radiation researchImprovement of a control stage. In Proceedings of the 2017 International Symposium on Space Technology and Science, Matsuyama, Japan, 3-9 June 2017.

Takahashi, A., Ikeda, H. and Yoshida, Y. (2018) Role of high-linear energy transfer radiobiology in space radiation exposure risks. Int. J. Part. Ther., 5, 151-159.

Takahashi, A., Yamanouchi, S., Takeuchi, K., Takahashi, S., Tashiro, M., Hidema, J., Higashitani, A., Adachi, T., Zhang, S., Guirguis, F., et al. (2020) Combined environment simulator for low-dose-rate radiation and partial gravity of Moon and Mars. Life (Base), 10, 274.

Wu, H., George, K., Willingham, V. and Cucinotta, F. A. (2001) Comparison of chromosome aberration frequencies in pre- and post-flight astronaut lymphocytes irradiated in vitro with gamma rays. Phys. Med., 17, 229-231.

Yamanouchi, S., Rhone, J., Mao, J. H., Fujiwara, K., Saganti, P. B., Takahashi, A. and Hada, M. (2020) Simultaneous exposure of cultured human lymphoblastic cells to simulated microgravity and radiation increases chromosome aberrations. Life (Basel), 10, 187.

Yang, T. C., George, K., Johnson, A. S., Durante, M. and Fedorenko, B. S. (1997) Biodosimetry results from space flight Mir-18. Radiat. Res., 148, S17-S23.

This article is licensed under a Creative Commons [Attribution 4.0 International] license. https://creativecommons.org/licenses/by/4.0/ 